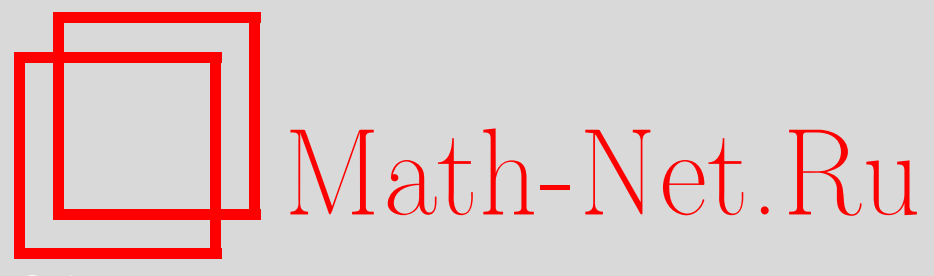

А. П. Розовская, Д. А. Шабанов, Об улучшении нижней оценки в задаче Косточки о полноцветных раскрасках гиперграфов, Матем. заметки, 2011, том 89, выпуск 6, 948-950

DOI: https://doi.org/10.4213/mzm9161

Использование Общероссийского математического портала Math-Net.Ru подразумевает, что вы прочитали и согласны с пользовательским соглашением http://www . mathnet.ru/rus/agreement

Параметры загрузки:

IP: 54.196 .121 .252

26 апреля 2023 г., 12:41:17

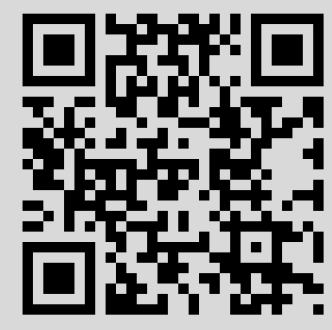




\section{Об улучшении нижней оценки в задаче Косточки о полноцветных раскрасках гиперграфов}

\section{А. П. Розовская, Д. А. Шабанов}

1. Введение и формулировка основного результата. В работе исследуется известная задача экстремальной теории гиперграфов. Напомним, что гиперграфом называется пара множеств $H=(V, E)$, где $V=V(H)$ есть некоторое конечное множество, называемое множеством вершин гиперграфа, а $E=E(H)$ есть совокупность каких-то подмножеств множества $V$, и эти подмножества называются ребрами гиперграфа. Гиперграф является $n$-равномерным, если каждое его ребро содержит ровно $n$ вершин.

Раскраска множества вершин гиперграфа в $r$ цветов $(r$-раскраска) называется полноиветной для $H$, если в ней каждое ребро из $E$ содержит вершины всех цветов. В работе Косточки [1] (см. также [2]) была поставлена задача об отыскании величины $p(n, r)$, равной минимальному числу ребер гиперграфа в классе $n$-равномерных гиперграфов, не имеющих полноцветных $r$-раскрасок, т.е.

$$
\begin{aligned}
p(n, r)=\min \{|E(H)|: H & -n \text {-равномерный гиперграф, } \\
& \text { для которого не существует полноцветных } r \text {-раскрасок }\} .
\end{aligned}
$$

Задача о нахождении величины $p(n, r)$ очень хорошо известна в случае $r=2$. Величина $p(n, 2)$ совпадает с классической величиной $m(n)$, равной минимальному количеству ребер $n$-равномерного гиперграфа, вершины которого нельзя покрасить в два цвета, так чтобы все ребра были неодноцветны. Задача об отыскании величины $m(n)$ была поставлена в 1961 г. Эрдешем и Хайналом в статье [3]. Наилучшие асимптотические оценки величины $m(n)$ были получены Эрдешем (верхняя оценка, [4]), а также Радхакришнаном и Сринивасаном (нижняя оценка, [5]):

$$
(\sqrt{3}-1)\left(\frac{n}{\ln n}\right)^{1 / 2} 2^{n-1} \leqslant p(n, 2)=m(n) \leqslant \frac{e \ln 2}{4} n^{2} 2^{n}(1+o(1)) .
$$

Косточкой были получены соотношения для произвольного $r$. Используя связь между полноцветными $r$-раскрасками $n$-равномерных гиперграфов и предписанными раскрасками полных $r$-дольных графов, он обосновал (см. [1]) следующие оценки для $p(n, r)$ при $r \geqslant 2$ и $n \geqslant 2$ :

$$
\frac{1}{r} e^{c_{1}(n / r)} \leqslant p(n, r) \leqslant r e^{c_{2}(n / r)},
$$

где $c_{1}$ и $c_{2}$ - некоторые абсолютные положительные константы, причем $c_{1}<1<c_{2}$.

Шабановым были получены (см. [6]) асимптотические верхние и нижние оценки $p(n, r)$ для случая $r=3$, которые затем были обобщены в работе [7] на случай произвольного значения $r$. Нижняя оценка выполняется для всех $n, r \geqslant 2, r<n$, и имеет вид

$$
p(n, r) \geqslant \frac{\sqrt{21}-3}{4 r}\left(\frac{n}{(r-1)^{2} \ln n}\right)^{1 / 3}\left(\frac{r}{r-1}\right)^{n} .
$$

Результат (3) асимптотически улучшает предыдущую оценку (2) при условии $r^{2}=O(n / \ln n)$ (подробнее, см. [7]). Верхняя оценка из работы [7] была получена в следующей области

Работа первого автора выполнена при поддержке гранта Президента РФ (грант № МК3429.2010.1). Работа второго автора выполнена при поддержке Российского фонда фундаментальных исследований (грант № 09-01-00294), Программы "Ведущие научные школы" (грант № НШ-8784.2010.1) и гранта Президента РФ (грант № МK-3429.2010.1). 
значений функции $r=r(n)$ : если $r=O\left(n^{2 / 3}\right)$, то

$$
p(n, r) \leqslant \frac{1}{r}\left(\frac{r}{r-1}\right)^{n} e(\ln r) \frac{n^{2}+\sqrt{n^{4}+16 n^{3} r(r-1)}}{4(r-1)} \varphi,
$$

где $\varphi$ - некоторая функция от $n$ и $r(n)$, стремящаяся к единице при $n \rightarrow \infty$. Результат (4) остается асимптотически наилучшим в области $r=O\left(n^{2 / 3}\right)$.

Основным результатом данной работы является новая нижняя оценка величины $p(n, r)$.

Теорема 1. Для всех $n \geqslant 3, r \geqslant 2$ таких, что $r<n /(2 \ln n)$, выполнено

$$
p(n, r) \geqslant \frac{1}{4 r}\left(\frac{n}{(r-1)^{2} \ln n}\right)^{1 / 2}\left(\frac{r}{r-1}\right)^{n} .
$$

Нетрудно видеть, что данный результат асимптотически улучшает предыдущие результаты (2) и (3) при условии $r^{2}=r^{2}(n)=o(n / \ln n)$. При этом оценка (5) улучшает (3) при $(r-1)^{2} \leqslant(\sqrt{21}-3)^{-6} n / \ln n$. Отметим также, что в классическом случае $r=2$ наша оценка (5) имеет тот же порядок роста, что и наилучшая нижняя оценка для $m(n)=p(n, 2)$ из (1), принадлежащая Радхакришнану и Сринивасану. Кроме того, с помощью (5) можно получить новые оценки числа вершин в полных многодольных графах с большим предписанным хроматическим числом (подробнее, см. [7]).

2. Условие существования полноцветной раскраски для реберных степеней. Одно из первых достаточных условий существования полноцветной $r$-раскраски в общем случае было получено Эрдешем и Ловасом в их совместной работе [8]. Обозначим через $\Delta(H)$ максимальную степень ребра гиперграфа $H$. Напомним, что степенъю ребра называется число других ребер гиперграфа, пересекающих данное ребро. В этих обозначениях теорема Эрдеша и Ловаса звучит следующим образом.

Теорема 2 (Эрдеш, Ловас, [8]). Если для п-равномерного гиперграфа Н выполнено

$$
\Delta(H) \leqslant \frac{r^{n-1}}{4(r-1)^{n}},
$$

то для $Н$ существует полноцветная г-раскраска.

Улучшение теоремы 2 было получено Шабановым в [7].

Теорема 3 (Шабанов, [7]). Пусть $r \leqslant n /(8 \ln n) u H-n$-равномерный гиперграф с условием

$$
\Delta(H) \leqslant \frac{\sqrt{12}-3}{8 r}\left(\frac{n}{(r-1)^{2} \ln n}\right)^{1 / 3}\left(\frac{r}{r-1}\right)^{n} .
$$

Тогда для Н существует полночветная $r$-раскраска.

Мы получаем дополнительное улучшение теоремы 3, ослабляя ограничение на максимальную степень ребра гиперграфа.

Теорема 4. Пусть даны произвольные натуральные числа $n \geqslant 3 u r \geqslant 2$, причем $r<n /(2 \ln n)$. Если п-равномерный гиперграф Н удовлетворяет условию

$$
\Delta(H) \leqslant \frac{\sqrt{13}-3}{32 r}\left(\frac{n}{(r-1)^{2} \ln n}\right)^{\frac{1}{2}}\left(\frac{r}{r-1}\right)^{n}-1,
$$

то для $Н$ существует полноцветная г-раскраска.

Если $r^{2}=o(n / \ln n)$, то ограничение $(6)$ на $\Delta(H)$ в теореме 4 , очевидно, асимптотически в $\left(n /\left((r-1)^{2} \ln n\right)\right)^{1 / 6}$ раз лучше ограничения на $\Delta(H)$ в теореме 3. Результат теоремы 4 может найти применение, например, в абстрактной теории автоматов (подробнее, см. [9]). 
3. Идеи доказательств. Доказательства теорем 1 и 4 основаны на применении метода случайной перекраски вершин гиперграфа. Мы приведем алгоритм построения случайной $r$-раскраски, с помощью дальнейшего вероятностного анализа которого и были получены оценки (5) и (6).

Пусть $H=(V, E)$ - произвольный $n$-равномерный гиперграф, а $\sigma$ - случайная нумерация множества $V$ с равномерным распределением. Сначала покрасим каждую вершину гиперграфа случайным образом: независимо от других в один из $r$ цветов с вероятностью $1 / r$. Обозначим получившуюся случайную равномерную раскраску через $\xi$. Построим на ее основе другую раскраску, применив следующий алгоритм случайной перекраски:

1) рассматриваем вершины $H$ по одной, согласно нумерации $\sigma$, т.е. на $i$-м шаге рассматриваем такую вершину $v$, что $\sigma(v)=i$;

2) если для вершины $v$ выполнены оба следующих условия:

а) вершина $v$ принадлежит ребру $e$, которое содержит вершины ровно $r-1$ цвета в раскраске $\xi$ (пусть, без ограничения общности, в $е$ отсутствует только цвет $\alpha$ ) и

b) ни на одном из предыдущих шагов ни одна вершина из $e$ не перекрасилась в цвет $\alpha$,

то перекрашиваем вершину $v$ следующим образом: с вероятностью $p$ в один из $r-1$ цветов, отличных от начального цвета $v$, и с вероятностью $1-(r-1) p$ вообще не перекрашиваем ее. Обозначим получившуюся после рассмотрения всех вершин случайную раскраску через $\chi$.

Оставшаяся работа состоит в том, чтобы обосновать тот факт, что для некоторого значения параметра $p$ с положительной вероятностью $\chi$ является полноцветной $r$-раскраской для $H$, если выполнено ограничение либо на число ребер (теорема 1 ), либо на максимальную степень ребра (теорема 4).

\section{СПИСОК ЦИТИРОВАННОЙ ЛИТЕРАТУРЫ}

[1] A. Kostochka, Electron. J. Combin., 9:1 (2002), Paper No. 9. [2] A. Kostochka, More Sets, Graphs and Numbers, Bolyai Soc. Math. Stud., 15, Springer-Verlag, Berlin, 2006, 175-197. [3] P. Erdős, A. Hajnal, Acta Math. Acad. Sci. Hungar, 12:1-2 (1961), 87-123. [4] P. Erdős, Acta Math. Acad. Sci. Hungar, 15:3-4 (1964), 445-447. [5] J. Radhakrishnan, A. Srinivasan, Random Structures Algorithms, 16:1 (2000), 4-32. [6] Д. А. Шабанов, Изв. РАН. Сер. матем., 71:6 (2007), 183-222. [7] Д. А. Шабанов, Матем. сб., 201:4 (2010), 137-160. [8] P. Erdős, L. Lovász, Infinite and Finite Sets, v. II, Colloq. Math. Soc. Janos Bolyai, 10, North-Holland, Amsterdam, 1975, 609-627. [9] F. Gécseg, B. Imreh, A. Pluhár, J. Autom. Lang. Comb., 3:2 (1998), 77-84.

\section{А. П. Розовская}

им. М. В. Ломоносова

E-mail: anarozovski@gmail.com

\section{Д. А. Шабанов}

Московский государственный университет

им. М. В. Ломоносова

E-mail: gold-amber@yandex.ru 